\title{
Improved capacity of a monkey- tropic HIV-1 derivative to replicate in cynomolgus monkeys with minimal modifications.
}

\section{$\operatorname{AUTHOR}(S)$ :}

Saito, Akatsuki; Nomaguchi, Masako; lijima, Sayuki; Kuroishi, Ayumu; Yoshida, Tomoyuki; Lee, Young-Jung; Hayakawa, Toshiyuki; ... Adachi, Akio; Matano, Tetsuro; Akari, Hirofumi

\section{CITATION:}

Saito, Akatsuki ... [et al]. Improved capacity of a monkey-tropic HIV-1 derivative to replicate in cynomolgus monkeys with minimal modifications.. Microbes and infection / Institut Pasteur 2011, 13(1): 58-64

\section{ISSUE DATE:}

2011-01

URL:

http://hdl.handle.net/2433/134587

\section{RIGHT:}

(C) 2010 Institut Pasteur Published by Elsevier Masson SAS; この論文は 著者最終稿です。内容が印刷版と異なることがありますので、引用の 際には出版社版をご確認ご利用ください。This is the Accepted Author Manuscript. Please cite only the published version. 


\title{
Improved capacity of a monkey-tropic HIV-1 derivative to replicate in cynomolgus monkeys with minimal modifications
}

\author{
Akatsuki Saito ${ }^{a}$ b, c, \#, Masako Nomaguchi ${ }^{\text {d, \#, Sayuki Iijima }}{ }^{c}$, Ayumu Kuroishi ${ }^{\mathrm{e}}$, \\ Tomoyuki Yoshida ${ }^{b}$, Young-Jung Lee ${ }^{c}$, Toshiyuki Hayakawa ${ }^{b}$, Ken Kono ${ }^{e}$, Emi E \\ Nakayama $^{\mathrm{e}}$, Tatsuo Shioda ${ }^{\mathrm{e}}$, Yasuhiro Yasutomi ${ }^{\mathrm{c}}$, Akio Adachi ${ }^{\mathrm{d}}$, Tetsuro Matano ${ }^{\mathrm{a}}$, \\ Hirofumi Akari ${ }^{\text {b,c,* }}$
}

${ }^{a}$ International Research Center for Infectious Diseases, The Institute of Medical Science, The University of Tokyo, Tokyo 108-8639, Japan

${ }^{\mathrm{b}}$ Primate Research Institute, Kyoto University, Inuyama 484-8506, Japan

${ }^{c}$ Tsukuba Primate Research Center, National Institute of Biomedical Innovation, Tsukuba 305-0843, Japan

${ }^{\mathrm{d}}$ Department of Microbiology, Institute of Health Biosciences, The University of Tokushima Graduate School, Tokushima 770-8503, Japan

${ }^{\mathrm{e}}$ Department of Viral Infections, Research Institute for Microbial Diseases, Osaka University, Suita 565-0871, Japan

Keywords: HIV-1; Old World monkey; TRIM5 $\alpha$

\# A.S. and M.N. contributed equally to this work.

*Corresponding Author:

Hirofumi Akari, D.V.M., Ph.D.

Primate Reseach Institute, Kyoto University, Inuyama 484-8506, Japan

E-mail: akari@pri.kyoto-u.ac.jp

Tel 81-568-63-0440 Fax 81-568-63-0459 


\section{Abstract}

Human immunodeficiency virus type 1 (HIV-1) hardly replicates in Old World monkeys.

Recently, a mutant HIV-1 clone, NL-DT5R, in which a small part of gag and the entire vif gene are replaced with SIVmac239-derived ones, was shown to be able to replicate in pigtail monkeys but not in rhesus monkeys (RM). In the present study, we found that a modified monkey-tropic HIV-1 (HIV-1mt), MN4-5S, acquired the ability to replicate efficiently in cynomolgus monkeys as compared with the NL-DT5R, while neither NL-DT5R nor MN4-5S replicated in RM cells. These results suggest that multiple determinants may be involved in the restriction of HIV-1 replication in macaques, depending on the species of macaques. The new HIV-1mt clone will be useful for studying molecular mechanisms by which anti-viral host factors regulate HIV-1 replication in macaques. 


\section{Introduction}

Human immunodeficiency virus type $1(\mathrm{HIV}-1)$ productively infects only humans but not Old

World monkeys (OWM) such as cynomolgus macaques (CM) or rhesus macaques (RM),

whereas RM-derived simian immunodeficiency virus (SIVmac) can efficiently replicate in

OWM. Because of this species barrier, alternative monkey models using SIVmac or

simian/human immunodeficiency viruses (SHIV) have been used for AIDS research [1-4].

However, detailed analyses of molecular mechanisms of the pathogenesis of HIV-1 have been

hampered by the lack of appropriate non-human primate models for HIV-1 infection.

The mechanistic basis for the inability of HIV-1 to replicate in OWM cells has remained

unclear. Recently, a number of intrinsic anti-HIV-1 cellular factors, including tripartite motif

protein $5 \alpha$ (TRIM5 $\alpha$ ), Cyclophilin A (CypA), apolipoprotein B mRNA-editing catalytic

polypeptide (APOBEC3) family and Tetherin were discovered in OWM cells $[5,6]$. TRIM5 $\alpha$

strongly suppresses HIV-1 replication, mainly by affecting the viral disassembly step,

resulting in a decrease of reverse transcription products $[7,8]$. CypA acts as a regulator

promoting TRIM5 $\alpha$-mediated restriction of HIV-1 [8]. APOBEC3 is also a major regulator of

HIV-1 replication $[9,10]$. APOBEC3 exerts its inhibitory effect mainly by inducing G to A

hypermutation into the viral genome due to its cytidine deaminase activity, while 
hypermutation-independent inhibitory activity at the stage of reverse transcription is also

evident [11]. Tetherin, also referred to as a BST-2, was identified as an intrinsic antiviral factor that restricts the egress of HIV-1 by tethering virions to the host cell surface $[12,13]$.

Importantly, HIV-1 can counteract human APOBEC3 activity by utilizing the viral accessory protein Vif, whereas it cannot counteract OWM APOBEC3 [14]. Similarly, HIV-1

counteracts human Tetherin activity by utilizing another viral accessory protein $\mathrm{Vpu}$, whereas HIV-1 does not counteract OWM Tetherin activity [15].

In an attempt to generate a monkey tropic HIV-1 (HIV-1mt), Kamada et al. constructed an HIV-1 variant carrying minimal SIVmac-derived sequences to overcome the restriction factors [16]. The prototype HIV-1 clone NL-DT5R had a sequence encoding an SIVmac loop between alpha-helices 4 and 5 (L4/5) of CA and the entire vif gene, which relieved the inhibitory effects on viral replication by restriction factors CypA, TRIM5 $\alpha$ and APOBEC3. NL-DT5R was able to replicate in pigtail monkeys (PM) in vivo as well as in vitro, as reported by Igarashi et al. [17]. Although NL-DT5R induced immune responses in infected animals, the virus did not establish persistent infection.

In the present study, we sought to adapt NL-DT5R to CM by performing long-term passage in CM-derived HSC-F cells. We successfully obtained a modified 
HIV-1mt clone having several mutations. Additionally, we inserted an SIVmac loop between alpha helices 6 and 7 (L6/7) of CA [18]. The resultant clone named MN4-5S was found to replicate efficiently and to induce strong immune responses in infected $\mathrm{CM}$, suggesting the impact of viral adaptation. 


\section{Materials and methods}

\subsection{Plasmid construction}

The HIV-1 derivatives were constructed on a background of an infectious molecular clone,

NL4-3 [19]. NL-DT5R, a cloned virus containing SIVmac239 L4/5 and the entire vif gene, was reported previously by Kamada et al. [16]. In addition, NL-DT562, a virus having an R5-tropic SF162-derived env gene on a background of NL-DT5R, was used in this study [20]. After long passage of NL-DT5R and NL-DT562 in cynomolgus T cell line HSC-F [21], several mutations were appeared in both viral genomes, and then all of them were inserted into NL-DT5R by gene-engineering techniques. Consequently, a clone having 14 nucleotide substitutions in its genome was constructed and named MN4-5. Among these substitutions, 7 were non-synonymous mutations. The structure of the clone is shown in Fig. 1. A part of L6/7 of CA (aa residues 120-122; HNP) of MN4-5 was also replaced with the corresponding segment of SIVmac239 CA (aa residues 120-123; RQQN) by means of site-directed mutagenesis as described previously [18]. The resultant construct was designated MN4-5S.

\subsection{Cells and viruses}


Human embryonic kidney cell line HEK293T was maintained in DMEM supplemented with $10 \%$ fetal bovine serum, 100 units $/ \mathrm{ml}$ of penicillin and $100 \mu \mathrm{g} / \mathrm{ml}$ of streptomycin (Sigma). Monkey peripheral blood mononuclear cells (PBMCs) were separated with a standard Ficoll density gradient separation method and cultured in R-10 composed of RPMI-1640 medium supplemented with $10 \%$ fetal bovine serum, 100 units $/ \mathrm{ml}$ of penicillin and $100 \mu \mathrm{g} / \mathrm{ml}$ of streptomycin (Sigma). The growth kinetics of each HIV-1 clone were examined in activated $\mathrm{CD}^{+}$cell-depleted PBMCs. Briefly, separated PBMCs were reacted with a PE-labeled anti-CD8 antibody and then treated with anti-PE magnetic beads. After washing, CD8 ${ }^{+}$ cell-depleted PBMCs were negatively separated by using MACS columns (Miltenyi Biotec). For stimulation, $\mathrm{CD}^{+}$cell-depleted PBMCs were first cultured in $\mathrm{R}-10$ containing $1 \mu \mathrm{g} / \mathrm{ml}$ of concanavalin A (Sigma) for 2 days followed by culture in R-10 supplemented with $100 \mathrm{U} / \mathrm{ml}$ IL-2 (Shionogi) for more 2 days. The cells were then infected with 100 ng of p24 of HIV-1 and the culture supernatant was collected periodically. HSC-F, a cynomolgus monkey-derived $\mathrm{CD}^{+} \mathrm{T}$ cell line [21], was cultured in R-10.

Virus stocks were prepared as follows: sub-confluent HEK293T cells were transfected with proviral DNA using Lipofectamine2000 reagent according to the manufacturer's instructions. At 42 hours after transfection, culture supernatants were centrifuged, filtrated with a $0.45 \mu \mathrm{m}$ 
filter, and aliquoted as virus stocks for in vitro experiments. For preparation of viral stocks for in vivo experiments, $\mathrm{CD}^{+}$cell-depleted PBMCs were infected with the HEK293T-derived stocks as described above. After washing, the cells were maintained for several days and the culture supernatants were collected and stored as described above.

\subsection{Reverse transcription (RT) assay}

Virion-associated RT activity was measured as described previously [22]. HSC-F cells $\left(1 \times 10^{6}\right)$ were infected with equal amounts of viruses ( $1 \times 10^{7} \mathrm{RT}$ units). Viral growth kinetics was determined by RT production in the culture supernatants.

\subsection{Animal experiments}

Healthy adult cynomolgus monkeys were used in this study. All animals were confirmed to be negative for simian retrovirus and were housed in individual isolators in a biosafety level 3 facility and maintained according to the National Institute of Biomedical Innovation rules and guidelines for experimental animal welfare. Bleeding and viral inoculation were performed under ketamine hydrochloride anesthesia. Viral stocks for inoculation were inoculated into each animal. The profiles of plasma viral RNA loads, circulating $\mathrm{CD}^{+}$and $\mathrm{CD}^{+} \mathrm{T}$ 
lymphocytes were evaluated as described below.

\subsection{Flow cytometry and immunophenotyping of peripheral blood lymphocytes}

Immunophenotyping of freshly isolated PBMCs was performed according to standard

procedures using multicolor flow cytometry performed with a FACSCantoII (Becton

Dickinson). $\mathrm{CD}^{+}$and $\mathrm{CD}^{+} \mathrm{T}$ cells were identified using monoclonal antibodies (mAbs) to

CD3 (clone SP34-2, BD Pharmingen), CD4 (clone L200, BD Pharmingen) and CD8 (clone

DK25, DAKO). Flow cytometric acquisition and analysis of samples was performed on at

least 10,000 events collected by a flow cytometer driven by FACSDiVa software.

\subsection{Analysis of antiviral antibody response}

Plasma samples from infected animals were first heat-inactivated at $56^{\circ} \mathrm{C}$ for $30 \mathrm{~min}$. Then,

100-fold diluted samples were reacted with commercially available anti-HIV-1 antibody detection strips (New LAV Blot I, Bio-Rad) according to the manufacture's instructions.

\subsection{In vivo depletion of $C D 8^{+}$lymphocytes}

Infected animals received an anti-CD8 mAb (cM-T807) as follows: $10 \mathrm{mg} / \mathrm{kg}$ (body weight) 
inoculation subcutaneously at 42 days post infection (DPI), followed by $5 \mathrm{mg} / \mathrm{kg}$ inoculation intravenously at 45, 49, and $52 \mathrm{DPI}$. The cM-T807 mAb was provided by the NIH Nonhuman Primate Reagent Resource. To repeatedly confirm the depletion of $\mathrm{CD}^{+}$cells in the presence of cM-T807, an anti-CD8 mAb (clone DK25, DAKO) was used as reported previously [23].

\subsection{Quantification of viral RNA}

Total RNA was collected from monkey plasma using a High Pure Viral RNA Kit (Roche Diagnostics) according to the manufacturer's instructions. Viral RNA was quantified with a quantitative real-time PCR system using TaqMan One-Step RT-PCR Master Mix Reagents (Applied Biosystems). The primers and probe used in this study were as follows: Forward primer: HIVgag683 (+) (5'-CTCTCGACGCAGGACTCGGCTTGCT-3'); Reverse primer: HIVgag803 (-) (5'-GCTCTCGCACCCATCTCTCTCCTTCTAGCC-3'); Probe: HIVgag TaqMan 720R748 (FAM- GCAAGAGGCGAGRGGCGGCGACTGGTGAG-TAMRA). The quantification and data analysis were performed using the iQ5 Real-Time PCR Detection System (Bio-Rad). The detection limit of this assay was 400-copies/ml plasma. 


\section{Results}

3.1. Growth properties of prototype HIV-1mt clone, NL-DT5R in macaques in vitro and in vivo

We first examined the replication properties of prototype HIV-1mt NL-DT5R in CD8 ${ }^{+}$ cell-depleted PBMCs of CM and RM. NL-DT5R replicated in the cells of CM but not in those of RM (Fig. 2). We next examined the in vivo replication properties of NL-DT5R in CM. Viral stocks for inoculation were prepared with $\mathrm{CD}^{+}$cell-depleted CM PBMCs as described above. Then, two monkeys were infected with NL-DT5R intravenously and bled periodically. As shown in Fig. 3A, NL-DT5R established infection as indicated by detectable levels of plasma viremia and an anti-HIV-1 antibody response, although the viral level was marginal (about $1 \times 10^{3}$ copies $/ \mathrm{ml}$ ) and disappeared at 4 weeks post infection. These results indicated that although CM appeared permissive for NL-DT5R as compared with RM, the mutations introduced in NL-DT5R were not still sufficient to overcome the restriction by host factor(s) of these macaques. 
In order to improve the replication capability of HIV-1mt in CM, we conducted long-term passage of NL-DT5R in HSC-F cells. Additionally, NL-DT562, having an R5-tropic env gene on a background of NL-DT5R, was also passaged long-term in HSC-F cells. We found that the passaging improved the growth of the viruses (data not shown), and then viral clones were obtained after the long-term passaging and sequenced. Ten nucleotide substitutions were indentified in the NL-DT5R-derived clone and 4 nucleotide substitutions (except for the env gene) in the NL-DT562-derived clone. These 14 nucleotide substitutions (7 of which were non-synonymous mutations) were assembled and introduced into NL-DT5R. The resultant clone was named MN4-5, and its structure is shown in Fig. 1. We previously found that insertion of an SIVmac loop between alpha helices 6 and 7 (L6/7) of CA into the corresponding region in HIV-1 significantly enhanced the viral replication in HSC-F cells and PBMCs of CM by relieving the inhibitory effect of TRIM5 $\alpha$ [18]. We therefore inserted an SIVmac-derived L6/7 sequence into MN4-5. The resultant clone was named MN4-5S (Fig. 1). In order to examine the impact of these modifications on the viral replication, we analyzed the replication properties of this "adapted" virus in HSC-F cells and CD8 ${ }^{+}$cell-depleted PBMCs of CM. MN4-5 showed higher replication as compared with NL-DT5R in both types of cells (Figs. 4 and 5). Moreover, MN4-5S showed enhanced growth capability in the cells as 
compared with the parental clones, NL-DT5R and MN4-5 (Figs. 4 and 5).

Notably, MN4-5S did not show any replication in RM cells (data not shown), indicating that the combination of the mutations introduced in NL-DT5R may be effective for escape from the restriction in CM cells but not in RM cells.

\subsection{MN4-5S induced greater viremia in CM as compared with parental clone, NL-DT5R}

Since MN4-5S showed enhanced ability to replicate in CM cells, we next examined the viral replication in vivo. The stock of MN4-5S virus was inoculated into $3 \mathrm{CM}$. MN4-5S induced 10-fold higher viremia in infected animals at 2-3 weeks after infection (Fig. 6A), as compared with that induced by NL-DT5R (see Fig. 3). This result was consistent with the in vitro result (Fig. 5) and demonstrated that the mutations inserted into NL-DT5R contributed to enhancement of viral replication in vivo. In addition, at the acute phase of infection a slight decrease of $\mathrm{CD}^{+} \mathrm{T}$ cells was observed (Fig. 6B). The viremia became undetectable at 6 weeks after infection. Thereafter, antibody response against MN4-5S was observed in infected animals (Fig. 6C). As indicated by comparison with the lane of the positive control as a standard, the degree of antibody response seemed to be stronger than that against NL-DT5R (see Fig. 3B and Fig. 6C). Next we attempted to clarify the role of $\mathrm{CD} 8^{+}$lymphocytes in the 
disappearance of viremia. We conducted in vivo depletion of $\mathrm{CD}^{+}$cells by using a method reported previously [23]. We found that the reappearance of viremia was observed in all monkeys tested in parallel with the decline of $\mathrm{CD}^{+} \mathrm{T}$ cells after the anti-CD8 mAb administration (Fig. 6, A and D). This result indicated that $\mathrm{CD}^{+} \mathrm{T}$ cells had a critical role in the control of HIV-1mt replication and suggested that the virus was able to infect latently in vivo. 


\section{Discussion}

In the present study, we found that a modified HIV-1mt, MN4-5S, acquired greater ability to replicate in CM than NL-DT5R, and that both the SIVmac-derived L6/7 (HNP120-122 > RQQN120-123 of CA) and a series of substitutions identified by long-term passage of NL-DT5R in HSC-F cells contributed to this ability (Fig.1). We recently showed that the substitution of L6/7 relieved the inhibitory effect of TRIM5 $\alpha$ [18]. Additionally, our preliminary data suggest that non-synonymous mutations in the integrase and env genes are likely to be critical for the improved activity (Nomaguchi et al., manuscript in preparation). It is possible that these adaptive mutations may optimize the interaction between host and viral proteins.

It seemed that the growth kinetics of NL-DT5R in PM were comparable with those of MN4-5S in CM, which had peak levels in acute viremia of approximately $10^{4}$ copies $/ \mathrm{ml}$ [17]. It is therefore likely that PM may exhibit better susceptibility to HIV-1mt than CM. It is possible that the greater susceptibility of PM to HIV-1mt replication could be due to the genotype of TRIM5, because PM usually express a chimera between TRIM5 $\alpha$ and CypA, so-called TRIM-Cyp, whose anti-HIV-1 activity is defective [24]. 
One unexpected finding in this study was that MN4-5S was unable to replicate in PBMCs of RM (data not shown), which was in contrast with the greater susceptibility of RM to SIVmac infection. Our results suggested that RM was most resistant to HIV-1mt replication among the three macaque species. Since our HIV-1mt clones (NL-DT5R and MN4-5) were established on the basis of information obtained from serial passages of the viruses in CM-derived cells, it may be reasonable to consider that these viruses were consequently optimized to CM. Alternatively, it is also possible that anti-HIV-1 activities such as TRIM5 $\alpha$ and APOBEC3 of RM could be greater than those of other macaques. Further studies are in progress to address these questions.

We demonstrated that the reappearance of viremia was observed in all monkeys tested in parallel with decline of $\mathrm{CD}^{+} \mathrm{T}$ cells after anti-CD8 mAb administration (Fig. 6, A and D). This result indicated that HIV-1-specific $\mathrm{CD}^{+} \mathrm{T}$ cells had a critical role in the control of HIV-1mt replication and suggested that the virus may be able to infect latently in vivo. In order to establish a set point viremia and persistent infection, further modifications of HIV-1mt may be required to enable potent escape from the antiviral immune response.

Further mechanistic characterization of anti-HIV-1 restriction factors will help in the construction of highly replicative and pathogenic HIV-1mt clones. As in the case of SHIV, 
in vivo passage of the virus could be a conventional and straightforward procedure for achieving such purposes [4]. However, the results of our study demonstrate that selective modification of HIV-1mt based on available knowledge regarding the molecular machineries is an alternative and powerful way. We are now in the process of developing the next generation of HIV-1mt that will acquire growth ability and pathogenicity in macaques as well as in humans. 


\section{Acknowledgements}

The authors wish to thank T. Kurosawa, M. Fujita and M. Yasue for their helpful assistance.

The authors also thank F. Ono, Y. Katakai, K. Komatsuzaki, A. Hiyaoka, K. Ohto, H. Ohto, and Y. Emoto for their support in animal experiments. We also thank M. Kaizu for his technical support. The anti-CD8 antibody used in the present study was provided by the NIH Nonhuman Primate Reagent Resource (R24 RR016001, N01 AI040101). This work was supported by grants from the Japan Health Sciences Foundation and the Ministry of Health, Labor, and Welfare in Japan and by Global COE Program A06 of Kyoto University. 


\section{References}

[1] N.L. Letvin, M.D. Daniel, P.K. Sehgal, R.C. Desrosiers, R.D. Hunt, L.M. Waldron, J.J. MacKey, D.K. Schmidt, L.V. Chalifoux, N.W. King, Induction of AIDS-like disease in macaque monkeys with T-cell tropic retrovirus STLV-III, Science 230 (1985) 71-73.

[2] H. Kestler, T. Kodama, D. Ringler, M. Marthas, N. Pedersen, A. Lackner, D. Regier, P. Sehgal, M. Daniel, N. King, et al., Induction of AIDS in rhesus monkeys by molecularly cloned simian immunodeficiency virus, Science 248 (1990) 1109-1112.

[3] R. Shibata, M. Kawamura, H. Sakai, M. Hayami, A. Ishimoto, A. Adachi, Generation of a chimeric human and simian immunodeficiency virus infectious to monkey peripheral blood mononuclear cells, J Virol 65 (1991) 3514-3520.

[4] K.A. Reimann, J.T. Li, R. Veazey, M. Halloran, I.W. Park, G.B. Karlsson, J. Sodroski, N.L. Letvin, A chimeric simian/human immunodeficiency virus expressing a primary patient human immunodeficiency virus type 1 isolate env causes an AIDS-like disease after in vivo passage in rhesus monkeys, J Virol 70 (1996) 6922-6928.

[5] M. Nomaguchi, N. Doi, K. Kamada, A. Adachi, Species barrier of HIV-1 and its jumping by virus engineering, Rev Med Virol 18 (2008) 261-275.

[6] D. Sauter, A. Specht, F. Kirchhoff, Tetherin: holding on and letting go, Cell 141 (2010) 392-398.

[7] M. Stremlau, C.M. Owens, M.J. Perron, M. Kiessling, P. Autissier, J. Sodroski, The cytoplasmic body component TRIM5alpha restricts HIV-1 infection in Old World monkeys, Nature 427 (2004) 848-853.

[8] E.E. Nakayama, T. Shioda, Anti-retroviral activity of TRIM5 alpha, Rev Med Virol 20 (2010) 77-92.

[9] A.M. Sheehy, N.C. Gaddis, J.D. Choi, M.H. Malim, Isolation of a human gene that inhibits HIV-1 infection and is suppressed by the viral Vif protein, Nature 418 (2002) 646-650.

[10] S. Henriet, G. Mercenne, S. Bernacchi, J.C. Paillart, R. Marquet, Tumultuous relationship between the human immunodeficiency virus type 1 viral infectivity factor (Vif) and the human APOBEC-3G and APOBEC-3F restriction factors, Microbiol Mol Biol Rev 73 (2009) 211-232.

[11] M.H. Malim, APOBEC proteins and intrinsic resistance to HIV-1 infection, Philos Trans R Soc Lond B Biol Sci 364 (2009) 675-687. 
S.J. Neil, T. Zang, P.D. Bieniasz, Tetherin inhibits retrovirus release and is antagonized by HIV-1 Vpu, Nature 451 (2008) 425-430.

[13] J.L. Douglas, J.K. Gustin, K. Viswanathan, M. Mansouri, A.V. Moses, K. Fruh, The great escape: viral strategies to counter BST-2/tetherin, PLoS Pathog 6 (2010) e1000913.

[14] R. Mariani, D. Chen, B. Schrofelbauer, F. Navarro, R. Konig, B. Bollman, C. Munk, H. Nymark-McMahon, N.R. Landau, Species-specific exclusion of APOBEC3G from HIV-1 virions by Vif, Cell 114 (2003) 21-31.

[15] B. Jia, R. Serra-Moreno, W. Neidermyer, A. Rahmberg, J. Mackey, I.B. Fofana, W.E. Johnson, S. Westmoreland, D.T. Evans, Species-specific activity of SIV Nef and HIV-1 Vpu in overcoming restriction by tetherin/BST2, PLoS Pathog 5 (2009) e1000429.

[16] K. Kamada, T. Igarashi, M.A. Martin, B. Khamsri, K. Hatcho, T. Yamashita, M. Fujita, T. Uchiyama, A. Adachi, Generation of HIV-1 derivatives that productively infect macaque monkey lymphoid cells, Proc Natl Acad Sci U S A 103 (2006) 16959-16964.

[17] T. Igarashi, R. Iyengar, R.A. Byrum, A. Buckler-White, R.L. Dewar, C.E. Buckler, H.C. Lane, K. Kamada, A. Adachi, M.A. Martin, Human immunodeficiency virus type 1 derivative with $7 \%$ simian immunodeficiency virus genetic content is able to establish infections in pig-tailed macaques, J Virol 81 (2007) 11549-11552.

[18] A. Kuroishi, A. Saito, Y. Shingai, T. Shioda, M. Nomaguchi, A. Adachi, H. Akari, E.E. Nakayama, Modification of a loop sequence between alpha-helices 6 and 7 of virus capsid (CA) protein in a human immunodeficiency virus type $1(\mathrm{HIV}-1)$ derivative that has simian immunodeficiency virus (SIVmac239) vif and CA alpha-helices 4 and 5 loop improves replication in cynomolgus monkey cells, Retrovirology 6 (2009) 70.

[19] A. Adachi, H.E. Gendelman, S. Koenig, T. Folks, R. Willey, A. Rabson, M.A.

Martin, Production of acquired immunodeficiency syndrome-associated retrovirus in human and nonhuman cells transfected with an infectious molecular clone, J Virol 59 (1986) 284-291.

[20] T. Yamashita, N. Doi, A. Adachi, M. Nomaguchi, Growth ability in simian cells of monkey cell-tropic HIV-1 is greatly affected by downstream region of the vif gene, J Med Invest 55 (2008) 236-240.

[21] H. Akari, K.H. Nam, K. Mori, I. Otani, H. Shibata, A. Adachi, K. Terao, Y. Yoshikawa, Effects of SIVmac infection on peripheral blood CD4+CD8+ T lymphocytes in cynomolgus macaques, Clin Immunol 91 (1999) 321-329.

[22] R.L. Willey, D.H. Smith, L.A. Lasky, T.S. Theodore, P.L. Earl, B. Moss, D.J. Capon, M.A. Martin, In vitro mutagenesis identifies a region within the envelope gene of the human immunodeficiency virus that is critical for infectivity, J Virol 62 (1988) 139-147. 
[23] J.E. Schmitz, M.A. Simon, M.J. Kuroda, M.A. Lifton, M.W. Ollert, C.W. Vogel, P. Racz, K. Tenner-Racz, B.J. Scallon, M. Dalesandro, J. Ghrayeb, E.P. Rieber, V.G. Sasseville, K.A. Reimann, A nonhuman primate model for the selective elimination of CD8+ lymphocytes using a mouse-human chimeric monoclonal antibody, Am J Pathol 154 (1999) 1923-1932.

[24] C.A. Virgen, Z. Kratovac, P.D. Bieniasz, T. Hatziioannou, Independent genesis of chimeric TRIM5-cyclophilin proteins in two primate species, Proc Natl Acad Sci U S A 105 (2008) 3563-3568.

\section{Legends of figures}

Fig. 1. Structure of HIV-1mt clones used in this study. The positions of nucleotide mutations are indicated by arrows in this figure. Among nucleotide substitutions, the positions of non-synonymous mutations are indicated in red.

Fig. 2. Growth properties of the NL-DT5R in $C D 8^{+}$cell-depleted PBMCs from CM (A) and RM (B). The cells were infected with NL-DT5R and the viral replication was monitored by p24 antigen in the culture supernatants using a p24 quantitative ELISA kit. Animal identifications are indicated at the top of each panel. 
Fig. 3. Profiles of plasma viral RNA loads (A) and anti-HIV-1 antibody responses (B) in CM infected with NL-DT5R. Mf97-108 (open circles) and Mf97-070 (closed diamonds) were used in this study. Viral stocks for inoculation were prepared in $\mathrm{CD}^{+}$cell-depleted PBMCs, and then $6.1 \mathrm{ng}$ p24 of HIV-1 were inoculated into each animal. Commercially available diagnostic HIV-1 Western blotting strips were reacted with 100-fold diluted monkey plasma. Plasma from HIV-1-infected or uninfected individuals was used as a positive or a negative control, respectively.

Fig. 4. Growth properties of HIV-1mt in HSC-F cells. The cells were infected with a series of HIV-1mt derivatives. The viral replication was monitored by RT activity in the culture supernatants.

Fig. 5. Growth properties of HIV-1mt in $\mathrm{CD}^{+}$cell-depleted PBMCs from four CM. The cells were infected with a series of HIV-1mt derivatives. The viral replication was monitored by p24 antigen in the culture supernatants using a p24 quantitative ELISA kit. Animal identifications are indicated at the top of each panel. 
Fig. 6. Profiles of plasma viral RNA loads (A), circulating CD4 ${ }^{+}$T lymphocytes (B), anti-HIV-1 antibody responses (C) and circulating $\mathrm{CD}^{+} \mathrm{T}$ lymphocytes (D) in CM infected with HIV-1 derivatives. Viral stocks for inoculation were prepared in $\mathrm{CD} 8^{+}$cell-depleted PBMCs and then 10 ng of p24 of HIV-1 were inoculated into each animal. Commercially available diagnostic HIV-1 Western blotting strips were reacted with 100-fold diluted plasma of each monkey. Plasma from HIV-1 infected or uninfected individuals was used as a positive or negative control, respectively. The black arrow indicates the day of anti-CD8 mAb (cM-T807) inoculation. 
Fig.1
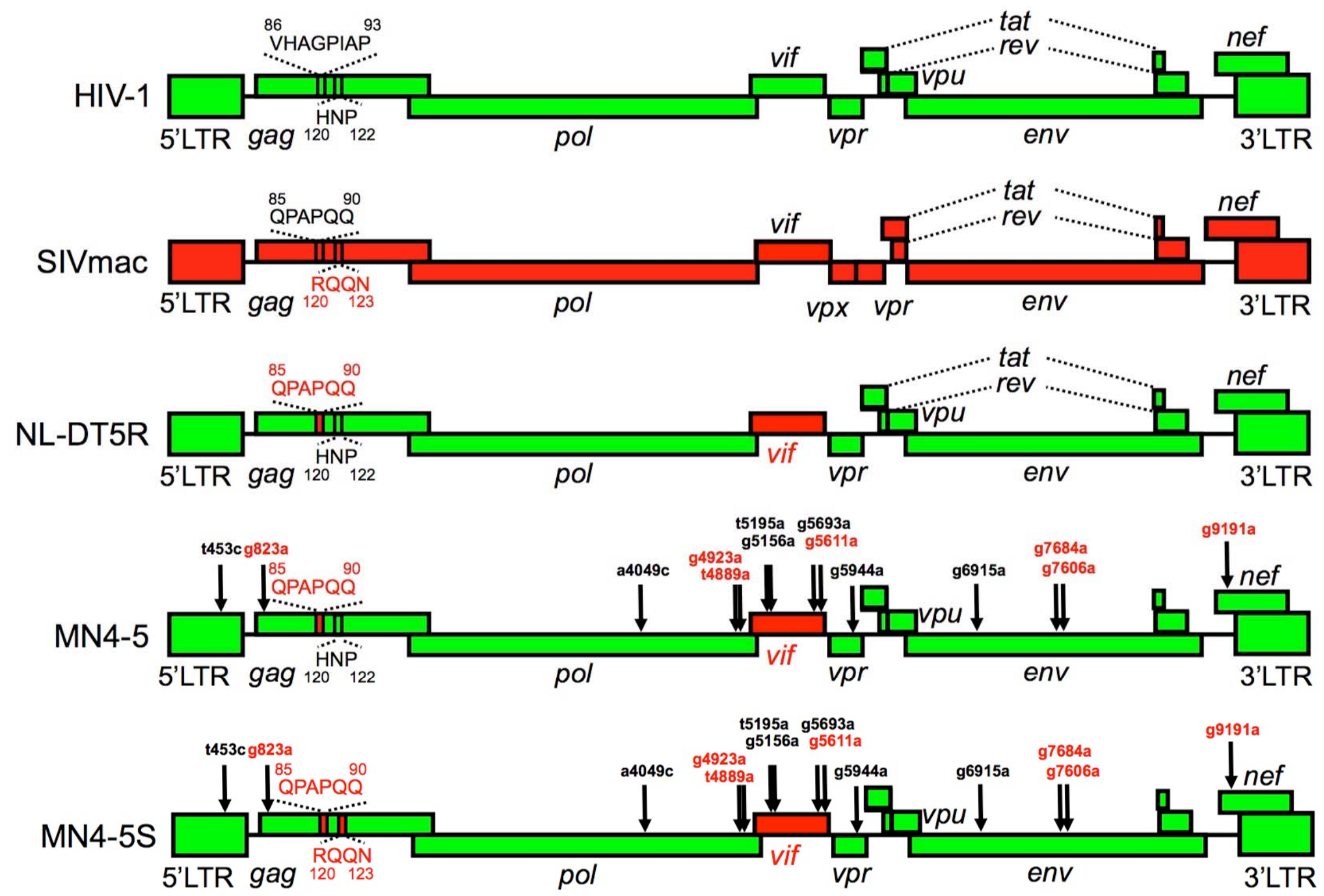
Fig.2
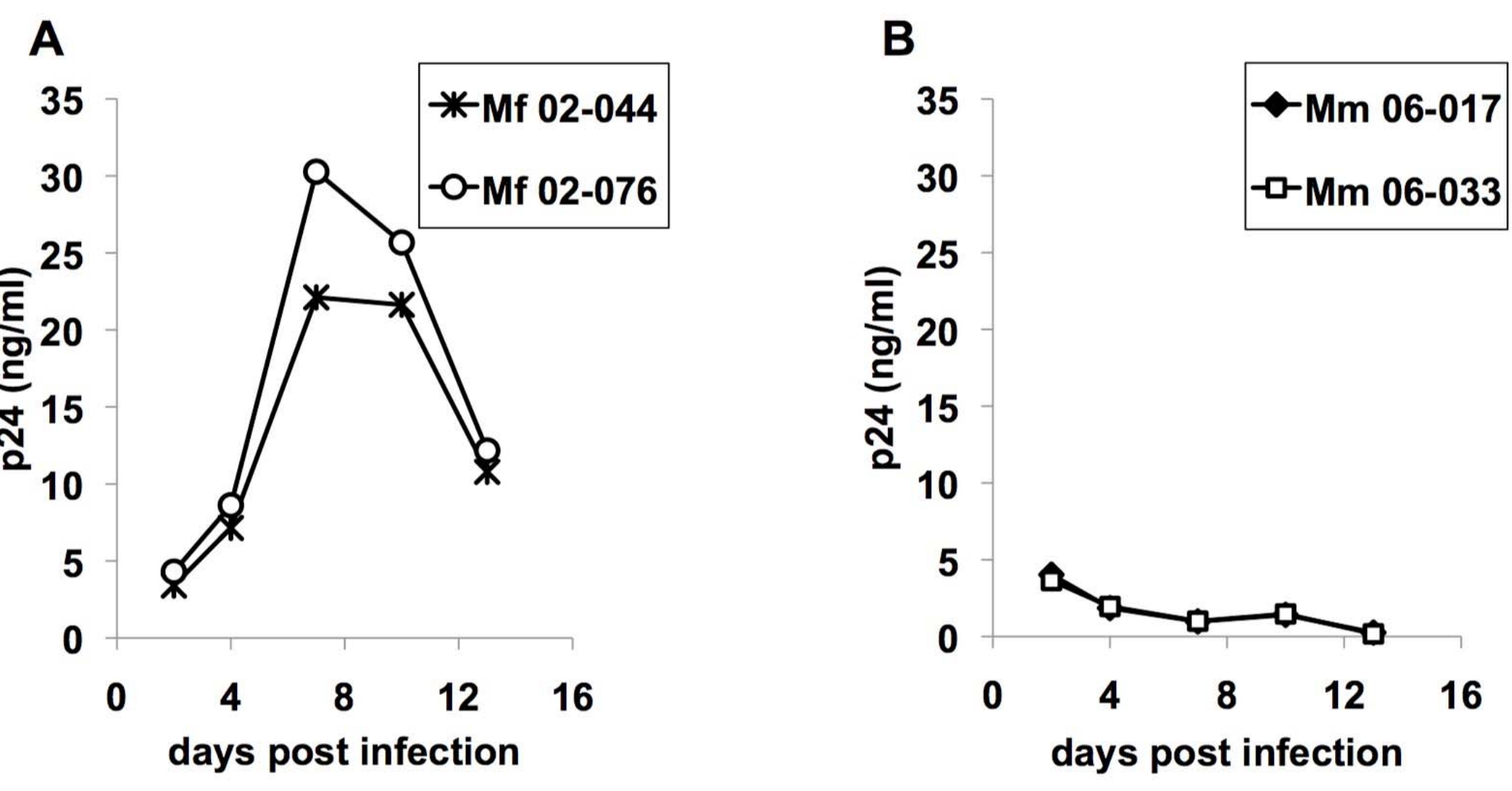
Fig.3
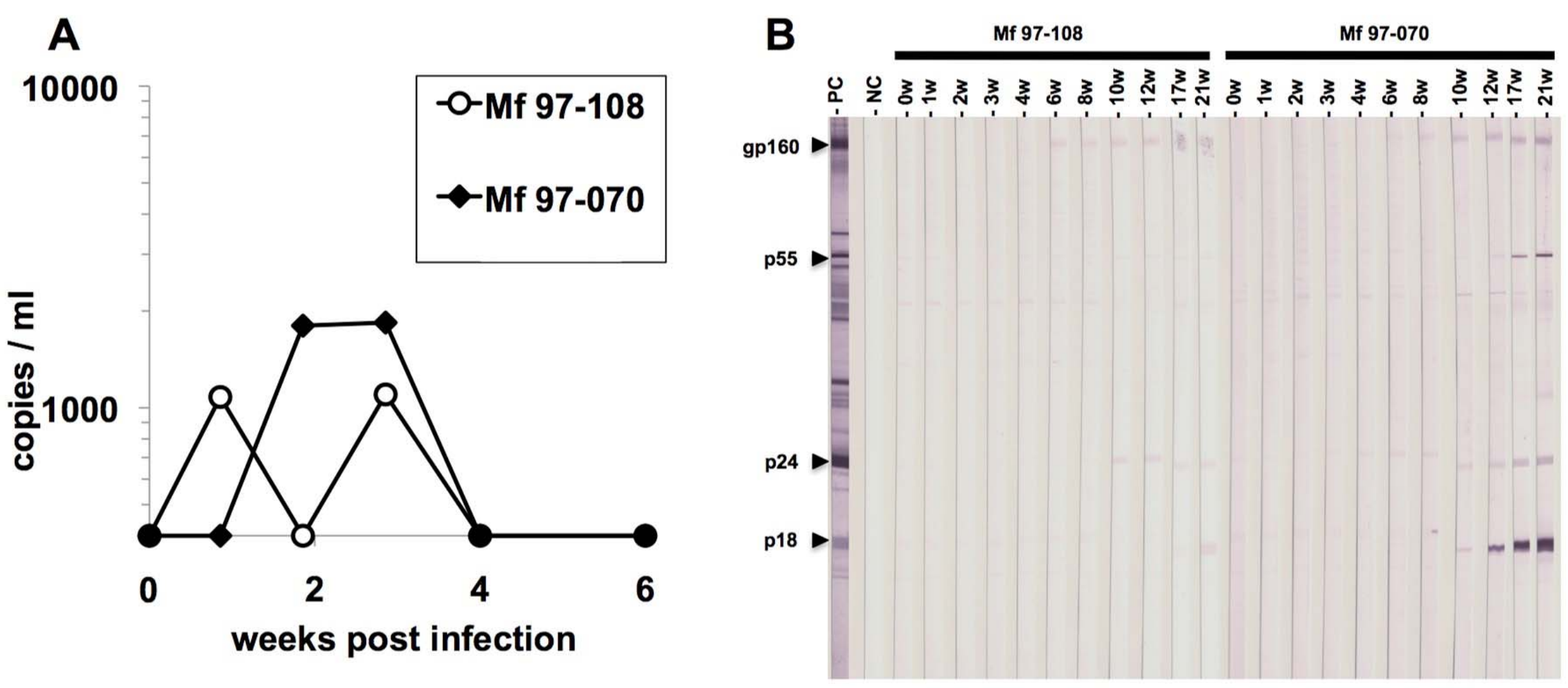
Fig.4

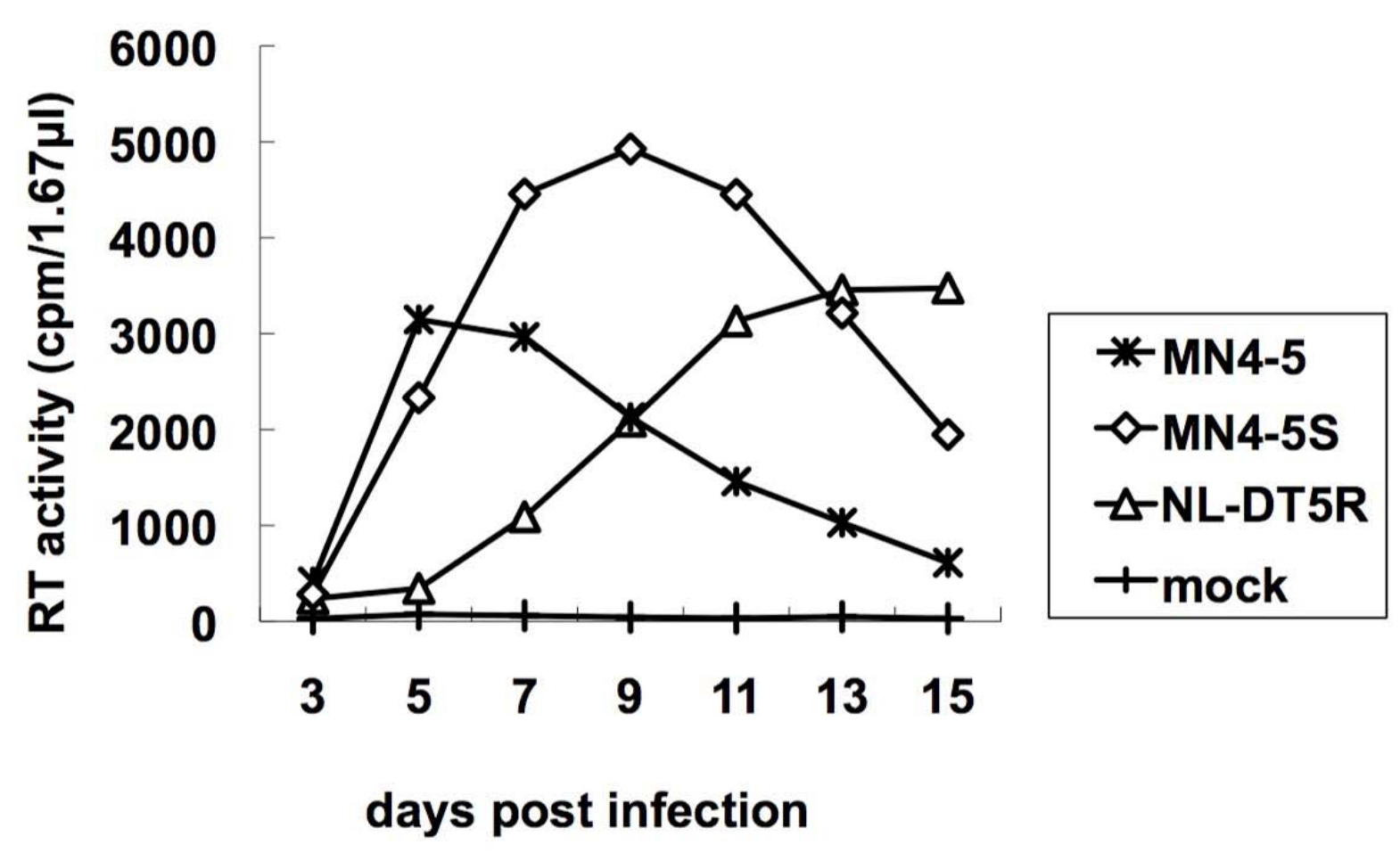


Fig.5
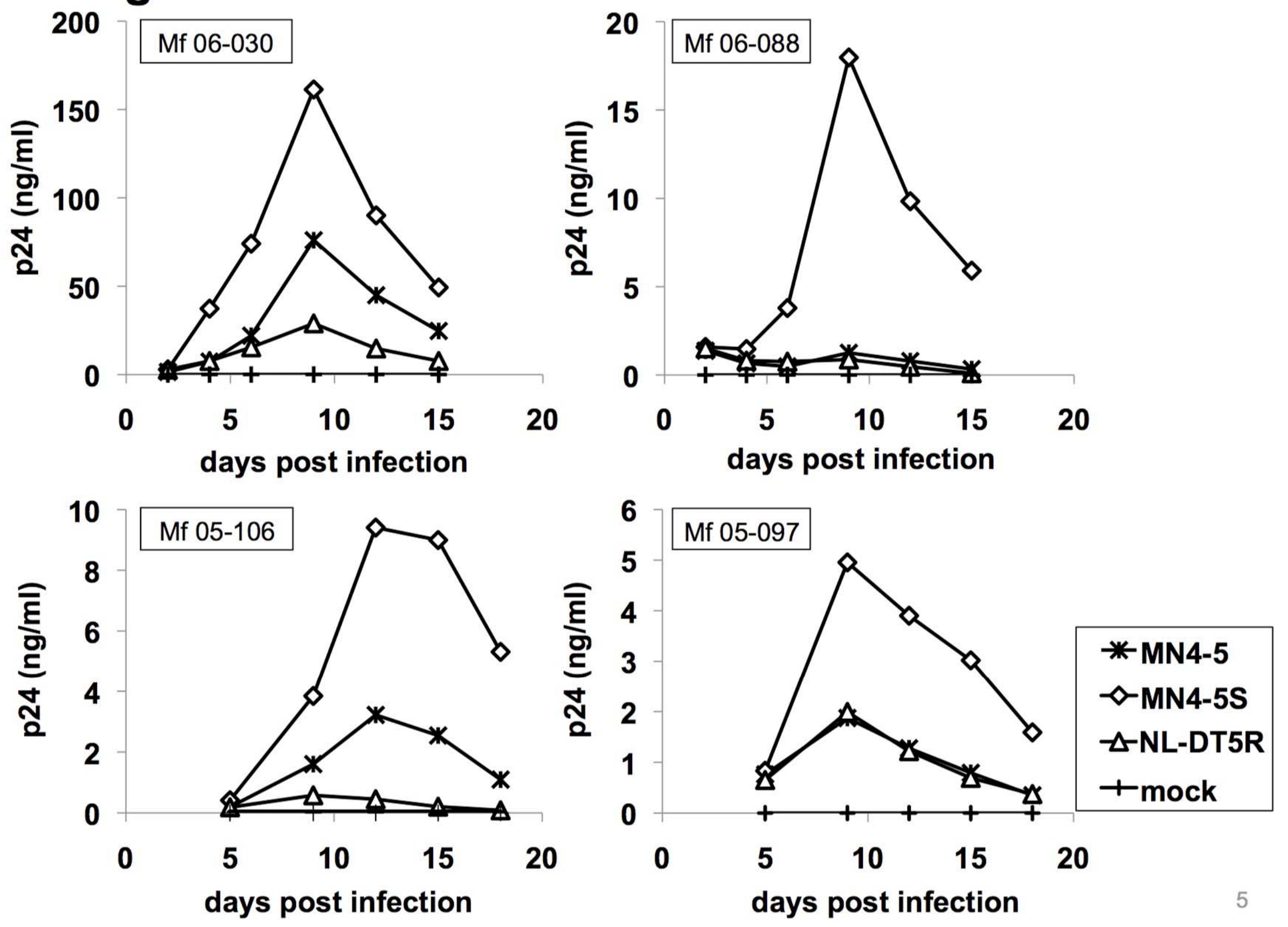
Fig.6

A

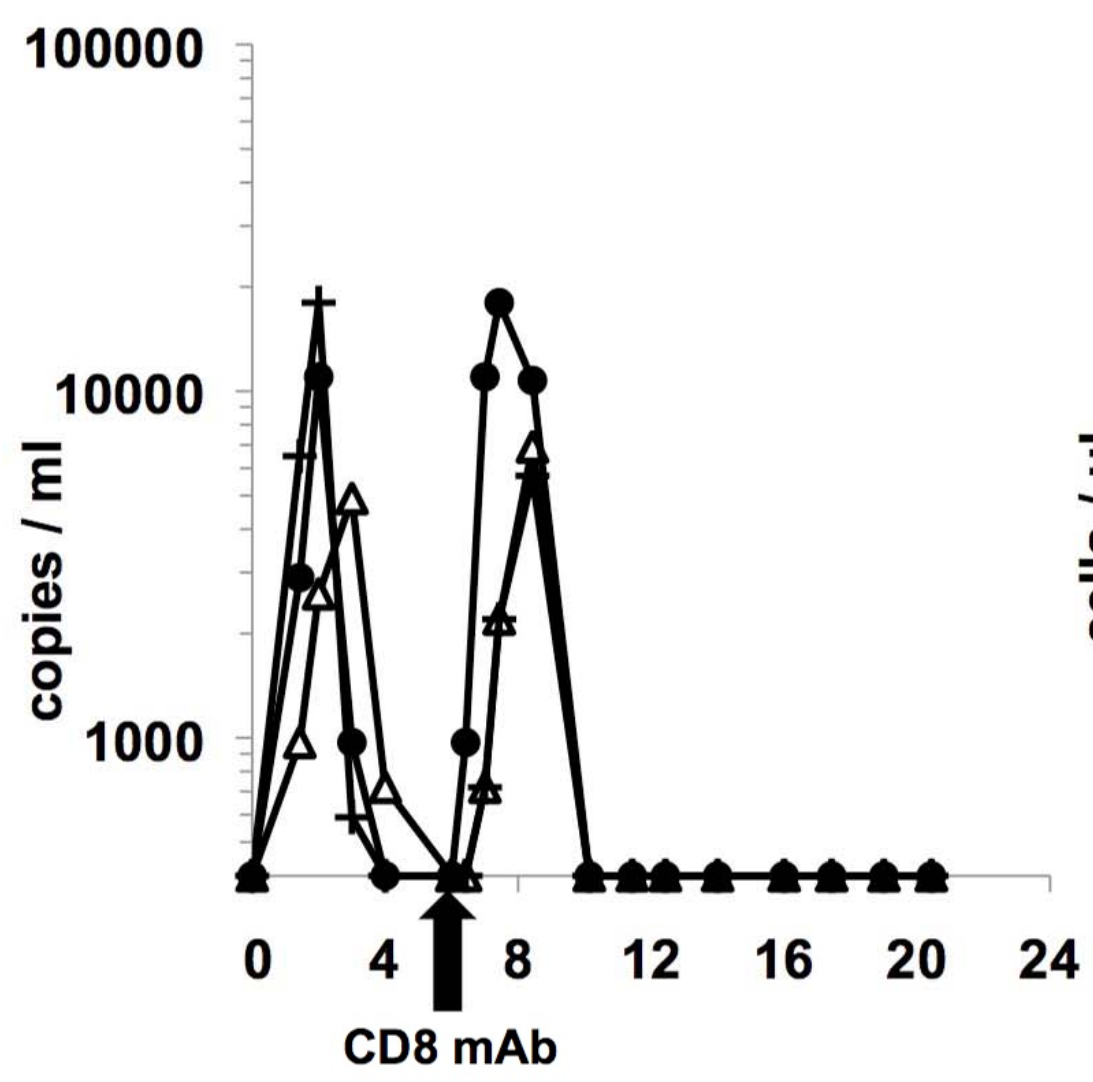

weeks post infection

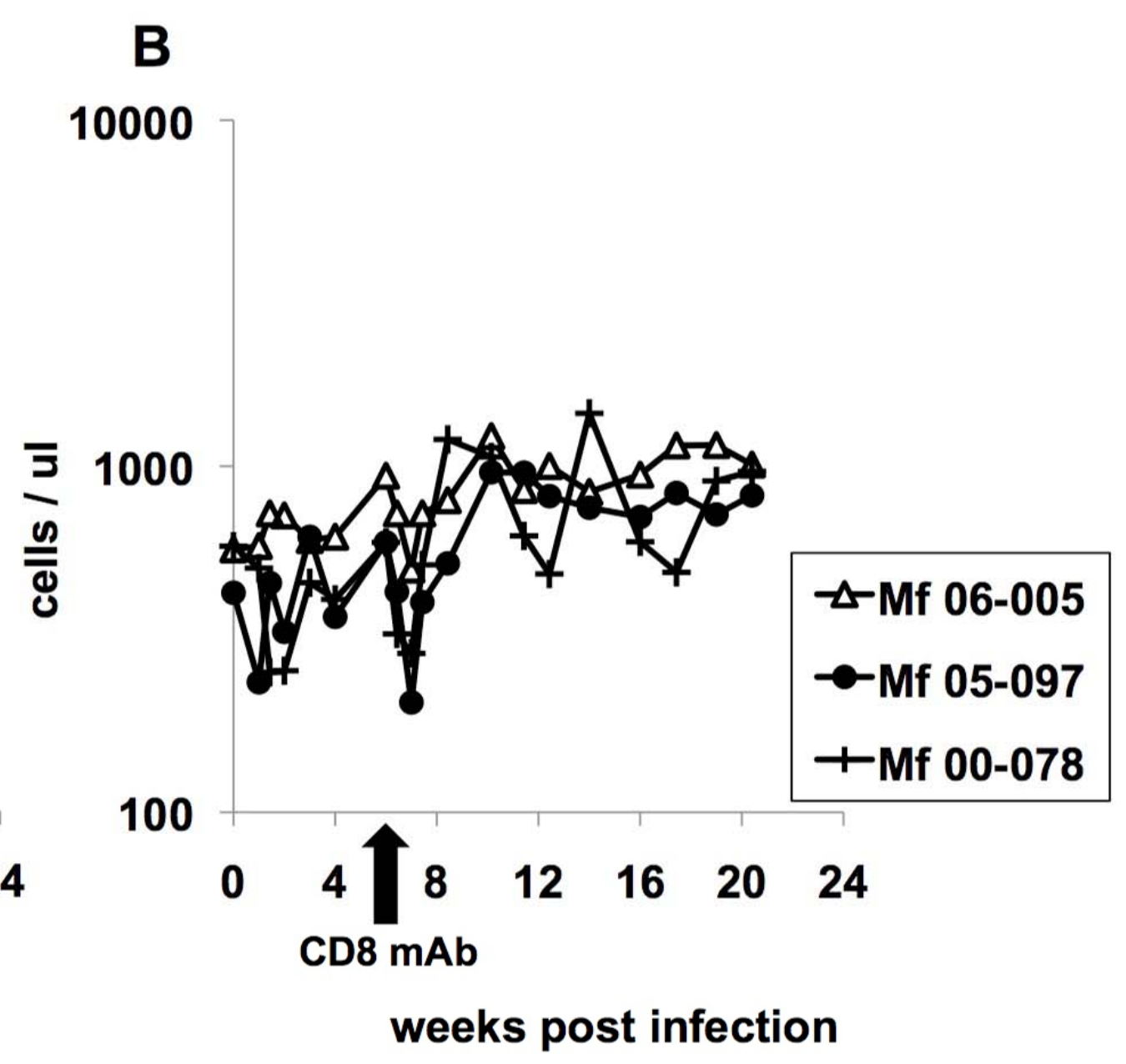



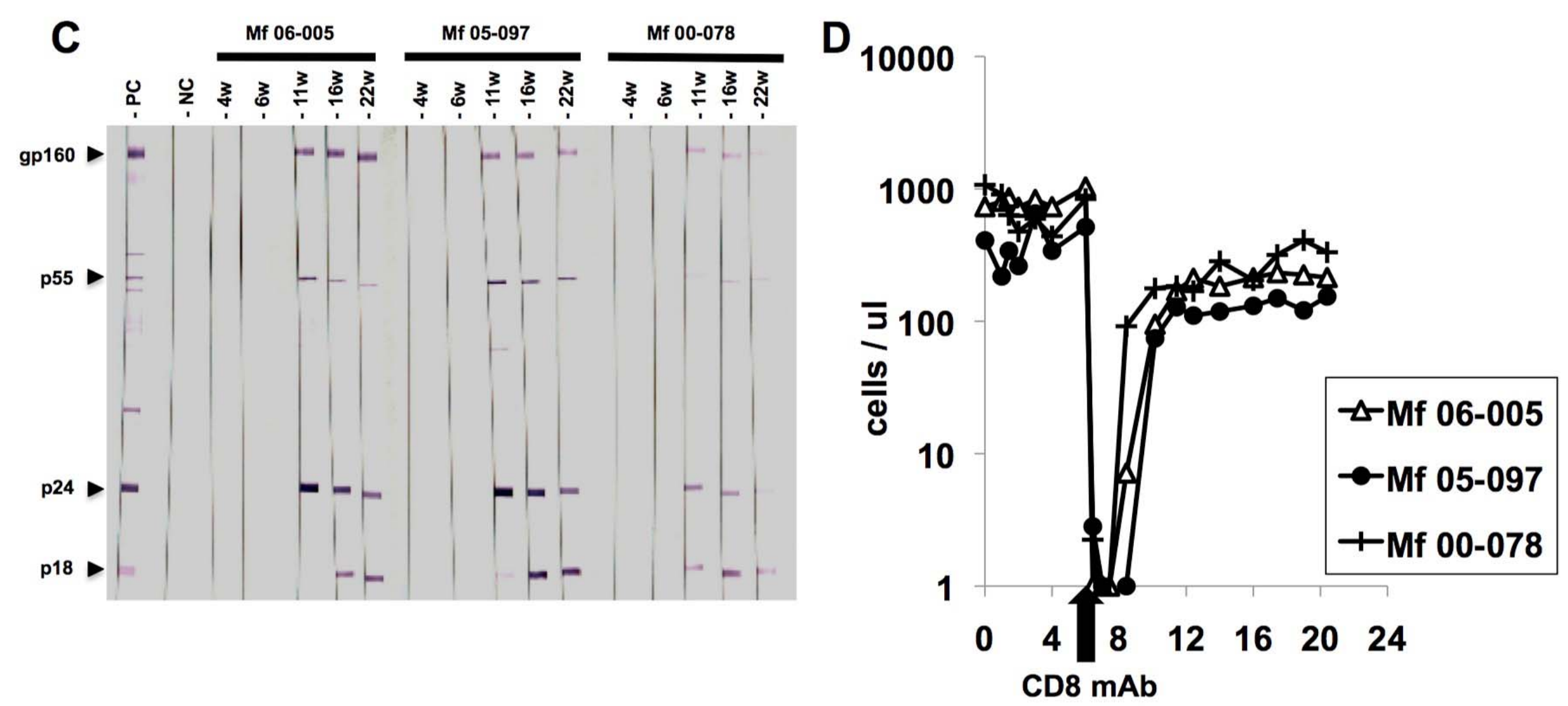

weeks post infection 